\title{
Association between interleukin 2 receptor $A$ gene polymorphisms (rs2104286 and rs12722489) with susceptibility to Multiple sclerosis in Iranian population
}

Majid Ahmadi ( $\sim$ Ahmadi.m@tbzmed.ac.ir)

Tabriz University of Medical Sciences https://orcid.org/0000-0003-4787-5261

Mojgan Hosseini

Islamic Azad University Eslamshahr Branch

Ramin pourakbari

Tabriz University of Medical Sciences

Saeed Aslani

Tehran University of Medical Sciences

Mohammad Hosein Ayoubi-joshaghani Ayoubi-joshaghani

Tabriz University of Medical Sciences

Bahareh Shirvani

Islamic Azad University Khorasgan Branch

Research article

Keywords: Multiple sclerosis, Gene polymorphism, IL-2RA

Posted Date: November 6th, 2019

DOI: https://doi.org/10.21203/rs.2.16898/v1

License: (c) (i) This work is licensed under a Creative Commons Attribution 4.0 International License.

Read Full License 


\section{Abstract}

Background: Multiple sclerosis (MS) is an organ-specific autoimmune disorder with remarkable heritability. For MS disorder, interleukin 2 receptor a subunit (IL2RA) is regarded as a genetic risk factor.

Results: There was a statistically significant association between alleles and genotypes of rs 12722489 SNP and MS risk. The levels of mRNA expression and serum IL-2RA were higher in MS patients than in healthy controls. mRNA expression and serum concentrations of IL-2RA were higher in MS patients with CC genotype for rs 12722489 compared with the rest of patients.

Conclusion: Our result demonstrate that the rs12722489 SNP within IL2RA gene might be associated with MS pathogenesis through regulating the levels of IL-2RA (or CD25), which is important in the regulation of T cells.

\section{Background}

Being a chronic disease, multiple sclerosis (MS) causes the central nervous system (CNS) to demyelinate (1). MS is principally diagnosed in adults who are in their twenties and thirties, and the frequency of the observation of this pathogenesis in women is higher than in men (2). According to the Multiple Sclerosis International Federation, MS affects almost 2.5 million people worldwide. Although many studies have been carried out on the subject, MS etiology has remained unidentified. Epidemiological data not only suggest that MS is an intricate disease, but they also demonstrate the role of environmental and genetic risk factors in its onset and progress (3). Hence, the human leukocyte antigen (HLA) locus may not describe the entire genetic constituent of this disease. Recently, non-HLA genetic risk factors have been recognized for MS, which have enticed remarkable attention for further investigations (4). Interleukin 2 receptor (IL-2R), especially a subunit (IL-2RA or CD25), is expressed on many immune cells, namely natural killer cells, activated $T$ cells and monocytes, as well as regulatory $T$ (Treg) cells (5).

Recent studies revealed that the soluble form of IL-2R subunit a (sIL-2RA) aggravated the MS conditions in an animal model not only by stimulating the differentiation of $\mathrm{T}$ helper (Th) 17 periphery cells, but also through augmenting the infiltration of both Th17 and Th1 cell subsets into the CNS. Moreover, the expression of the related orphan receptor gamma (RORYt), the critical transcription factor of Th17 cells, was induced by sIL-2RA, which also inhibited Signal transducer and activator of transcription 5 (STAT5), leading to the increased secretion of interleukin (IL)-17 (6). In addition, the homeostasis, proliferation, activation and differentiation of FoxP3 ${ }^{+}$Treg cells is much related on IL-2R function and $\mathrm{IL}-2$ signaling (7). Thus, the smallest polymorphism in CD25 gene region can lead to various diseases, especially autoimmune diseases, namely type 1 diabetes (T1D), multiple sclerosis (MS), Rheumatoid arthritis (RA), Grave's disease (GD), and Systemic lupus erythematosus (SLE)(8).

The estimated location of the human IL2RA gene is on chromosome 10 (10p15-p14). In the recent decade, the association of two common single nucleotide polymorphisms (SNPs) in the IL2RA gene (rs12722489 and rs2104286) has been widely evaluated with several autoimmune diseases (9). The 
association between MS susceptibility and SNPs in the IL2RA gene has been established in many countries, including Australia, India, Japan and United States (9-11), yet it has not been assessed in Iran. In this study, the rs 2104286 and rs 12722489 , as two SNPs in an intron of the IL2RA gene in an Iranian population were assessed for susceptibility to MS, and the impression and association of these variations in secretion of sIL-2RA, as well as its transcript level in PBMCs, were estimated.

\section{Study Population And Methods Patients and control subject}

A total of 230 MS patients ( 66 men and 164 women), who had been examined between 2017 and 2018 at Emam Reza Hospital, Tabriz, Iran, were enrolled. Utilizing the 2010 revised McDonald criteria for MS, the determination of MS was performed (12). Similarly, 280 healthy controls (177 men and 103 women) with no sign of inflammatory diseases before assessment were enrolled. The baseline characteristics of the participants is listed in Table 1. While all the subjects took the written informed consent, the Ethics Committee of Tabriz University of Medical Sciences, Tabriz, Iran, approved the protocol of this study. Likewise, this experiment was implemented according to The Code of experiments in humans. For experimenting, $5 \mathrm{ml}$ of peripheral blood was obtained from both patients and healthy control individuals.

\section{Genotyping of IL2RA gene polymorphisms}

Study participants were genotyped for the IL2RA gene rs2104286 and rs 12722489 polymorphisms using Real-time allelic discrimination TaqMan technique (Applied Biosystems, Foster City, USA). The amplification mixture included $5 \mu \mathrm{l}$ TaqMan Master Mix which itself contained Taq DNA polymerase and dNTPs (Applied Biosystems, Foster City, USA), $0.25 \mu$ TaqMan Genotyping Assay mix which contained primers as well as FAM or VIC labeled probes (Applied Biosystems, Foster City, USA), a total of $4.5 \mu \mathrm{l}$ of genomic DNA (20-30 $\mathrm{ng} / \mu \mathrm{l})$, and $0.25 \mu \mathrm{l}$ of $\mathrm{H}_{2} \mathrm{O}$ in the ultimate volume of $10 \mu \mathrm{l}$. Additionally, Real-time allelic discrimination PCR was performed utilizing Step One plus Real-time PCR system (Applied Biosystems, Foster City, USA). Furthermore, the thermocyclic considerations were the following: initial 60 ${ }^{\circ} \mathrm{C}$ for 30 seconds, and then $95^{\circ} \mathrm{C}$ for 10 minutes, then 40 amplification cycles $\left(95^{\circ} \mathrm{C}\right.$ for 15 seconds and $60^{\circ} \mathrm{C}$ for 1 minutes), and eventually $60^{\circ} \mathrm{C}$ for 30 seconds.

\section{RNA extraction and complementary DNA (CDNA) synthesis}

Total RNA extraction was accomplished through utilizing RNX-PLUS Solution on the cultured PBMCs (SinaClon, Tehran, Iran). Taking advantage of ratios of absorbance at 260/280 and 260/230, RNA quality and quantity were measured and assessed. In order to measure IL-2RA's expression levels, cDNA synthesis was implemented with oligo(dT) and random hexamer primers utilizing M-MLV (H-) Revert Aid Reverse Transcriptase kit (Thermo Fisher, Waltham, MA). 


\section{Real-time quantitativePCR of IL2RA gene expression}

Quantitative RT- PCR was performed using SYBER Green Master mix (Takara Biotechnology [Dalian] Co. Ltd) in the Light Cycler 2.0 Real-Time PCR System machine (Roche Applied Science, Germany). To normalize the expression of mRNA and miRNA, internal controls, RNU6 in particular, were included. Primers Sequences are summarized in Table 1.

\section{sIL-2RA concentration}

Enzyme-linked immunosorbent assay (ELISA) measurement of sIL-2RA was accomplished in accordance with the manufacturer's recommendations (BD Biosciences). Samples of serum origin were diluted 1:20 through consuming phosphate buffer saline (PBS). Reading Microtiter plates was performed utilizing a Biorad Benchmark microplate reader. Providing an outcome with a normal distribution was carried out via utilizing a log10 transformation of total sIL-2RA concentration.

\section{Statistical analysis}

To determine the association of alleles, genotypes, dominant and recessive inheritance models with the disorder risk, the chi-square test was exerted. Odds ratios (ORs) with $95 \%$ confidence intervals $(95 \% \mathrm{Cl})$ were also measured for each comparison. The Hardy-Weinberg Equilibrium (HWE) was calculated for the control group in each SNP. For all analysis, SHEsis online tool (http://shesis.bio-x.cn/SHEsis.html) and STATA software were used. The group comparisons for mRNA expression level as well as serum concentrations were performed by independent sample $t$ test or ANOVA. Pearson's correlation coefficient was conducted to perform correlation analysis. $P$ values less than 0.05 were considered as statistically significant level.

\section{Results}

\section{Allele and genotype frequencies}

Genotype frequencies for both rs2104286 and rs12722489 SNPs in control group did not diverge from $\operatorname{HWE}(P=0.478$ and 0.514 , respectively).

Genotypic results revealed that the C allele of rs 12722489 was proportionally related with a higher risk of MS (Table 2). Likewise, the analysis suggested a notable result in the $\mathrm{C} v s$. T, CC vs. TT, CT vs. TT, CC+CT vs. TT, and CC vs. CT+TT comparison in the overall population ( $\mathrm{OR}=1.85,95 \% \mathrm{Cl}: 1.34-2.56 . P<0.0002)$, $(\mathrm{OR}=4.87,95 \% \mathrm{Cl}: 1.55-15.26 P<0.0065),(\mathrm{OR}=1.71,95 \% \mathrm{Cl}: 1.16-2.53 . P<0.0063),(\mathrm{OR}=1.89,95 \% \mathrm{Cl}:$ 1.29-2.75. $P<0.0009)$, and $(\mathrm{OR}=4.13,95 \% \mathrm{Cl}: 1.32-12.85 . P<0.014)$, respectively (Table 2$)$.

Similarly, the results of genotyping showed that the A allele of rs2104286 was proportional to an elevated risk of MS (Table 3). In the same way, the analysis suggested a notable result in the AA vs. GG 
comparison in the overall population $(\mathrm{OR}=1.60,95 \% \mathrm{Cl}: 0.91-2.81, P<0.097)($ Table 3$)$.

\section{Gene expression}

The mRNA expression level of IL-2RA gene analysis showed that IL-2RA mRNA expression was higher in MS patient compared to the control group (Figure1.A). Also, the level of expression of a series of alleles in the two genotypic groups was different in patients compared with normal subjects $(1.023 \pm 0.015)$. In the rs12722489 genotype, the expression level of CC $(1.243 \pm 0.530 P=0.0001), C T(1.121 \pm 0.143 ; P=$ $0.0001)$, and TT (1.171 $\pm 0.33 ; P=0.0001)$ was remarkably increased (Figure 1.B). In addition, in the rs2104286 genotype the expression level of AA (1.202 $\pm 0.42 ; P=0.0001)$, AG $(1.090 \pm 0.18 ; P=0.0001)$, and GG $(1.167 \pm 0.21 ; P=0.0001)$ was notably increased (Figure 1.C).

\section{sIL-2RA concentration}

CD25 secretion level was measured by ELISA in the PBMCs' supernatant of the MS patients and control groups. Secretion of IL-2RA significantly increased in MS patients, compare to healthy people $(P=$ 0.0004; Figure 2.A). In addition, in this study, we investigated the level of CD25 secretion in rs 12722489 and rs2104286 groups, and the results revealed that: In the rs 12722489 genotype, TT vs. CT (1966.60 $\pm 843.80,2334.6 \pm 766.30 ; P=0.017)$ had significant differences (Figure 2.B), and in the rs2104286 genotype, AA vs. AG (1620.5 $\pm 432.7,2267.9 \pm 870.4 ; P=0.01)$, AA vs. GG (1620.5 \pm 432.7 , 2354.68 $\pm 937.8 ; P=0.0018)$, and GG vs. GG $(1845.35 \pm 875.7,2354.68 \pm 937.8 ; P=0.0002)$ had significant differences (Figure 2.C).

\section{Correlation analysis}

According to Table 4, the expression of IL-2RA mRNA was correlated significantly with EDSS in MS patients with CC genotype for rs $12722489(r=0.55, P=0.0021)$. Moreover, a significant correlation was observed in the serum levels of IL-2RA and EDSS in MS patients with CC genotype for rs12722489 ( $r=$ $0.61, P=0.0133)$.

\section{Discussion}

In spite of the notable contribution of HLA in MS disorder, there is a significant percentage of unknown genetic factors involved in MS pathogenesis. The association of two most common polymorphisms of the IL2RA gene (rs2104286 and rs12722489) with an elevated risk of MS has been evaluated in plenty of papers. However, the association of these genotypes with respect to the conflicting data is still unknown.

Being an integral membrane compound, the IL -2 receptor consists of various protein structures, such as $a$ (CD25), $\beta$ (CD122), and $Y$ (CD132). As mentioned, the signaling of the IL-2 receptor is of paramount importance for immune cell activation, especially those involved in the pathogenesis of MS disease, such 
as the T cell subtypes. Consequently, there is an opinion that inhibition of IL-2 signaling can inhibit T cells (13).

The rs2104286 and rs12722489 SNPs are located inside the primary intron of the IL2RA gene. However, these SNPs do not directly alter the IL2-RA structure, but rather can change the expression of IL2RA through influencing the utilities of the regulatory components, including messenger RNA (mRNA), and mRNA half-life (9).

In this study, it was realized that the A allele of rs2104286 was related to a higher risk of MS, which is similarly shown in previous studies including Japanese, Canadian and Indian patients (14-16). Likewise, the $C$ allele of rs 12722489 was related with an elevated risk of MS. However, the association of this allele with MS disease in some studies has not been seen yet (17).

In addition, the secretion level of this receptor was also associated with polymorphisms of rs2104286 A allele and rs12722489 C allele in MS patients compared with healthy subjects in this study. It is not clear how these polymorphisms can affect the sIL-2RA gene expression. In segregated studies, buhelt and Maier have demonstrated that the level of SCD25 is affected by the MS-associated SNP rs2104286 in the IL2RA gene $(9,18)$.

Recently, in the clinical phase, daclizumab as a human monoclonal antibody that attaches to IL-2RA, has been tested on MS patients. Furthermore, Clinical studies have revealed that injecting daclizumab subcutaneously to patients with MS that are in the process of recovery reduces the number of lesions in the brain's MRI. Daclizumab could increase the proliferation and improve the utility of regulatory NK cells, which possess the capability to decrease activated T cells (19).

In addition, Daclizumab causes a blockade to the action of IL-2 presentation via mature dendritic cells to primed T cells, in consequence, T cells and maybe other immune cells will be significantly reduced (20). Daclizumab also prevents the meningeal lymphoid agglomeration and correlates to the immune memory responses through hindering lymphoid tissue inducer (LTi) cells (21).

By investigating the effects of daclizumab, the role of $\mathrm{IL}-2$ in the pathogenesis of multiple sclerosis was clearly determined, and it is hoped that with further understanding of mutations and polymorphism of this receptor gene, a definite treatment for this autoimmune disease can be found.

In the end, some of our work restrictions need to be declared. Firstly, absence of accessibility to individual information restrained the assessment of feasible gene-gene and gene-environment interplay. Secondly, the relationship between the IL2RA polymorphisms and MS subtype such as Relapsing-remitting MS (RRMS) or primary progressive MS (PPMS) was not evaluated. Despite all these restrictions, the milestones of this study were the investigation of the level of gene expression as well as the measurement of the CD25 secretion with its relations to the aforementioned polymorphisms.

\section{Declarations}




\section{Acknowledgement}

The authors are grateful of Student's Research Committee, Tabriz University of Medical Sciences. Also the authors are grateful of the patients and the healthy individuals for their participation in the study.

\section{Authors' contributions}

RP: Performed the experiments, participated in manuscript drafting and read the manuscript. MH; Performed the statistical analysis, participated in manuscript drafting, and read the manuscript critically. SA; Participated in conducting the experiments and read the manuscript critically. MA: contributed to patient management and sample preparation and read the manuscript critically. MA; Developed the main idea, participated in manuscript drafting, and and read the manuscript critically. BS; Helped in the study design, participated in manuscript drafting, and and read the manuscript critically. All authors read and approved the final manuscript.

\section{Funding}

The financial support of this study was underwritten by a grant from Student's Research Committee, Tabriz University of Medical Sciences, Tabriz, Iran. Grant number: 98-217.

\section{Availability of data and materials}

The data that support the findings of this study are available on request from the corresponding author. The data are not publicly available due to privacy or ethical restrictions. All data generated or analyzed during this study are included in this published article.

\section{Ethics approval and consent to participate}

The protocol of this study was approved by the Human Research Ethics Committee from the Tabriz University of Medical Sciences, Tabriz, Iran and written informed consent forms was taken by all subjects.

\section{Consent for publication}

All authors read the manuscript and consent for its publication.

\section{Competing interests}

The authors declare that they have no competing interests. 


\section{References}

1.Koudriavtseva T, Mainero C: Neuroinflammation, neurodegeneration and regeneration in multiple sclerosis: intercorrelated manifestations of the immune response. Neural regeneration research 2016, 11(11):1727-1730.

2.Howard J, Trevick S, Younger DS: Epidemiology of Multiple Sclerosis. Neurologic clinics 2016, 34(4):919-939.

3.Hunter SF: Overview and diagnosis of multiple sclerosis. The American journal of managed care 2016, 22(6 Suppl):s141-150.

4.Jiang L, Bai X, Wang Y, Wei M: Association between CD24-P226-C/T polymorphism and multiple sclerosis: a meta-analysis. Immunological investigations 2015, 44(4):321-330.

5.Wang J, Wicker LS, Santamaria P: IL -2 and its high-affinity receptor: genetic control of immunoregulation and autoimmunity. Seminars in immunology 2009, 21(6):363-371.

6. Russell SE, Moore AC, Fallon PG, Walsh PT: Soluble IL-2Ralpha (SCD25) exacerbates autoimmunity and enhances the development of Th17 responses in mice. PloS one 2012, 7(10):e47748.

7.Bayer AL, Yu A, Malek TR: Function of the IL $-2 R$ for thymic and peripheral CD4+CD25+ Foxp3+ T regulatory cells. The Journal of Immunology 2007, 178(7):4062-4071.

8.0'Shea JJ, Ma A, Lipsky P: Cytokines and autoimmunity. Nature Reviews Immunology 2002, 2(1):37.

9.Maier LM, Lowe CE, Cooper J, Downes K, Anderson DE, Severson C, Clark PM, Healy B, Walker N, Aubin C: IL2RA genetic heterogeneity in multiple sclerosis and type 1 diabetes susceptibility and soluble interleukin-2 receptor production. PLoS genetics 2009, 5(1):e1000322.

10.Matiello M, Weinshenker BG, Atkinson EJ, Schaefer-Klein J, Kantarci OH: Association of IL2RA p olymorphisms with susceptibility to multiple sclerosis is not explained by missense mutations in IL2RA. Multiple Sclerosis Journal 2011, 17(5):634-636.

11.Qiu W, Pham K, James I, Nolan D, Castley A, Christiansen FT, Czarniak P, Luo Y, Wu J, Garlepp M: The influence of non-HLA gene polymorphisms and interactions on disease risk in a Western Australian multiple sclerosis cohort. Journal of neuroimmunology 2013, 261(1-2):92-97.

12.Polman CH, Reingold SC, Banwell B, Clanet M, Cohen JA, Filippi M, Fujihara K, Havrdova E, Hutchinson M, Kappos L: Diagnostic criteria for multiple sclerosis: 2010 revisions to the McDonald criteria. Annals of neurology 2011, 69(2):292-302.

13.Chistiakov DA, Voronova NV, Chistiakov PA: The crucial role of IL-2/IL-2RA-mediated immune regulation in the pathogenesis of type 1 diabetes, an evidence coming from genetic and animal model 
studies. Immunology letters 2008, 118(1):1-5.

14.Ainiding G, Kawano Y, Sato S, Isobe N, Matsushita T, Yoshimura S, Yonekawa T, Yamasaki R, Murai H, Kira J-i: Interleukin 2 receptor a chain gene polymorphisms and risks of multiple sclerosis and neuromyelitis optica in southern Japanese. Journal of the neurological sciences 2014, 337(1-2):147150.

15.Traboulsee AL, Bernales CQ, Ross JP, Lee JD, Sadovnick AD, Vilariño-Güell C: Genetic variants in IL2RA and IL7R affect multiple sclerosis disease risk and progression. Neurogenetics 2014, 15(3):165-169.

16.D'Cunha MA, Pandit L, Malli C: CD6 gene polymorphism rs17824933 is associated with multiple sclerosis in Indian population. Annals of Indian Academy of Neurology 2016, 19(4):491-494.

17.Consortium IMSG: Risk alleles for multiple sclerosis identified by a genomewide study. New England Journal of Medicine 2007, 357(9):851-862.

18.Buhelt S, Ratzer RL, Christensen JR, Bornsen L, Sellebjerg F, Sondergaard HB: Relationship between soluble CD25 and gene expression in healthy individuals and patients with multiple sclerosis. Cytokine 2017, 93:15-25.

19.Rose JW, Giovannoni G, Wiendl H, Gold R, Havrdova E, Kappos L, Selmaj KW, Zhao J, Riester K, Tsao LC et al: Consistent efficacy of daclizumab beta across patient demographic and disease activity subgroups in patients with relapsing-remitting multiple sclerosis. Multiple sclerosis and related disorders 2017, 17:32-40.

20.Wuest SC, Edwan JH, Martin JF, Han S, Perry JS, Cartagena CM, Matsuura E, Maric D, Waldmann TA, Bielekova B: A role for interleukin-2 trans-presentation in dendritic cell-mediated T cell activation in humans, as revealed by daclizumab therapy. Nature medicine 2011, 17(5):604-609.

21. Howell OW, Reeves CA, Nicholas R, Carassiti D, Radotra B, Gentleman SM, Serafini B, Aloisi F, Roncaroli $\mathrm{F}$, Magliozzi R et al: Meningeal inflammation is widespread and linked to cortical pathology in multiple sclerosis. Brain: a journal of neurology 2011, 134(Pt 9):2755-2771.

\section{Tables}

Table 1. The demographic features and clinical characteristics of the MS patients and control group

\begin{tabular}{|c|c|c|l}
\hline$P$ value & Controls & MS patients & Trait \\
\hline & 280 & 230 & Number \\
\hline 0.26 & $26-50(35.13 \pm 7.74)$ & $28-51(34.28 \pm 8.43)$ & Age (mean) \\
\hline 0.93 & $177 / 103$ & $164 / 66$ & Gender (Female/ Male) \\
\hline 0.41 & $24.2 \pm 3.9$ & $25.3 \pm 4.6$ & BMl \\
\hline & - & $1.85 \pm 0.92$ & EDSS \\
\hline & - & $2-6$ yrs. & Disease duration \\
\hline
\end{tabular}


Table 2. Allele and genotype frequencies of IL2RA gene rs2104286 and rs12722489 SNPs in MS patients and healthy controls and related association analyses.

\begin{tabular}{l|c|c|}
\hline \multirow{2}{*}{ dbSNP } & \multicolumn{2}{|c|}{ Frequency } \\
\cline { 2 - 3 } & MS patients (N=230) & Controls (N=280) \\
\hline rs2104286 & $159(34 \%)$ & $169(30 \%)$ \\
\hline A & $301(65 \%)$ & $391(70 \%)$ \\
\hline G & $34(14.8 \%)$ & $28(10 \%)$ \\
\hline AA & $91(39.6 \%)$ & $113(40.4 \%)$ \\
\hline GG & $105(45.7 \%)$ & $139(49.6 \%)$ \\
\hline
\end{tabular}

HWE for controls $=0.478$

Association Test

\begin{tabular}{l|l|l|l|l|}
\hline \multicolumn{2}{|l|}{ Model } & OR & $95 \%$ CI & $P$ value \\
\hline Allele & A vs. G & 1.22 & $0.93-1.59$ & 0.135 \\
\hline Codominant & AA vs. GG & 1.60 & $0.91-2.81$ & 0.097 \\
\hline Codominant & AG vs. TT & 1.06 & $0.73-1.55$ & 0.737 \\
\hline Dominant & AA+AG vs. TT & 1.17 & $0.82-1.66$ & 0.369 \\
\hline Recessive & AA vs. AG+TT & 1.56 & $0.91-2.66$ & 0.102 \\
\hline rs 12722489 & & & & \\
\hline C & $105(22.8 \%)$ & $77(13.8 \%)$ \\
\hline T & $355(77.2 \%)$ & & $483(86.3 \%)$ \\
\hline CC & $13(5.7 \%)$ & \multicolumn{3}{|c|}{$6(1.4 \%)$} \\
\hline CT & $79(34.3 \%)$ & $69(34.6 \%)$ \\
\hline TT & $138(60 \%)$ & $(73.9 \%)$ \\
\hline
\end{tabular}

HWE for controls $=0.514$

Association Test

\begin{tabular}{|l|l|c|c|c|}
\hline \multicolumn{2}{|l|}{ Model } & OR & $95 \%$ CI & $P$ value \\
\hline Allele & C vs. T & 1.85 & $1.34-2.56$ & 0.0002 \\
\hline Codominant & CC vs. TT & 4.87 & $1.55-15.26$ & 0.0065 \\
\hline Codominant & CT vs. TT & 1.71 & $1.16-2.53$ & 0.0063 \\
\hline Dominant & CC+CT vs. TT & 1.89 & $1.29-2.75$ & 0.0009 \\
\hline Recessive & CC vs. CT+TT & 4.13 & $1.32-12.85$ & 0.014 \\
\hline
\end{tabular}

*SNP; single nucleotide polymorphism, MS; Multiple sclerosis, OR; odds ratio, 95\% Cl; $95 \%$ confidence interval, HWE; HardyWeinberg equilibrium 
Table 3. Overall haplotype associations of the SNPs according to Haploview.

\begin{tabular}{|c|c|c|c|c|c|c|c|}
\hline \multicolumn{3}{|c|}{ Block 1 Haplotypes } & \multicolumn{2}{|c|}{ Haplotype Frequencies } & \multirow[t]{2}{*}{$\mathrm{OR}^{*}(95 \% \mathrm{Cl})$} & \multirow[t]{2}{*}{$x^{2}$} & \multirow[t]{2}{*}{$P$ value } \\
\hline Row & rs2104286 & rs12722489 & $\begin{array}{c}\text { MS } \\
\text { N (\%) }\end{array}$ & $\begin{array}{c}\text { (Control) } \\
\mathrm{N}(\%)\end{array}$ & & & \\
\hline 1 & A & C & $91.25(19.8 \%)$ & $70.88(12.7 \%)$ & $1.75(1.24-2.45)$ & 10.65 & 0.0011 \\
\hline 2 & $\mathrm{~A}$ & $\bar{T}$ & $67.75(14.7 \%)$ & $98.12(17.5 \%)$ & $0.83(0.59-1.16)$ & 1.47 & 0.284 \\
\hline 3 & G & C & $13.75(3 \%)$ & $6.12(1.1 \%)$ & - & - & \\
\hline 4 & G & $T$ & $287.25(62.4 \%)$ & $384.88(68.7 \%)$ & $0.79(0.60-1.03)$ & 2.93 & 0.08 \\
\hline
\end{tabular}

MS; Multiple sclerosis, OR; odds ratio, 95\% Cl; 95\% confidence interval

Table 4. Correlation analysis of disease severity (EDSS) with mRNA expression and serum levels of IL2RA.

\begin{tabular}{|l|c|c|}
\hline & $\begin{array}{c}\text { mRNA } \\
\boldsymbol{r}(\boldsymbol{P} \text { value })\end{array}$ & $\begin{array}{c}\text { Serum level } \\
\boldsymbol{r}(\boldsymbol{P} \text { value })\end{array}$ \\
\hline EDSS (rs12722489 / CC) & $0.55(0.0021)$ & $0.61(0.0133)$ \\
\hline EDSS (rs12722489 / CT) & $0.21(0.086)$ & $0.19(0.48)$ \\
\hline EDSS (rs12722489 / TT) & $0.11(0.44)$ & $0.25(0.61)$ \\
\hline Total patients & $0.29(0.16)$ & $0.31(0.21)$ \\
\hline
\end{tabular}

\section{Figures}

A.

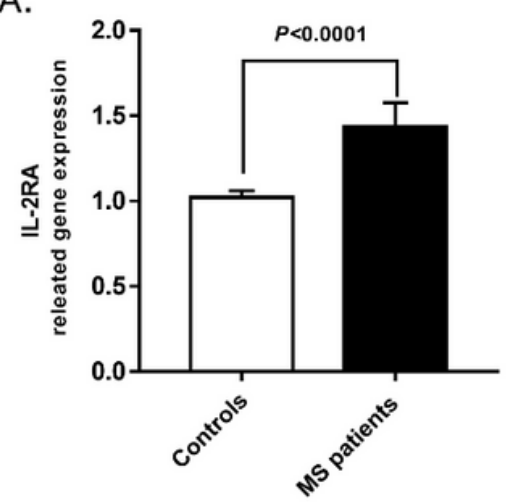

B.

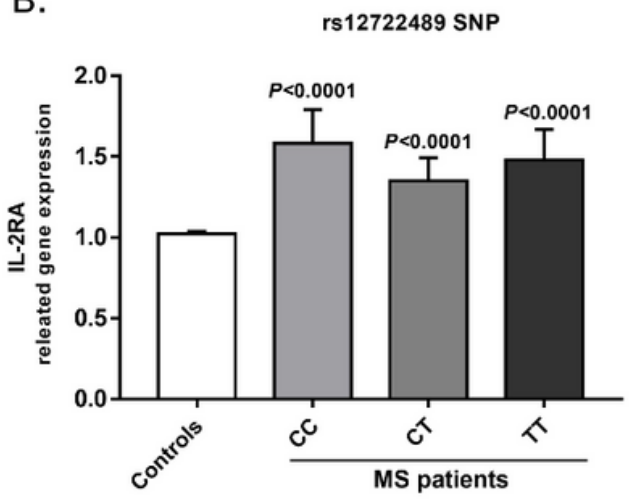

C.

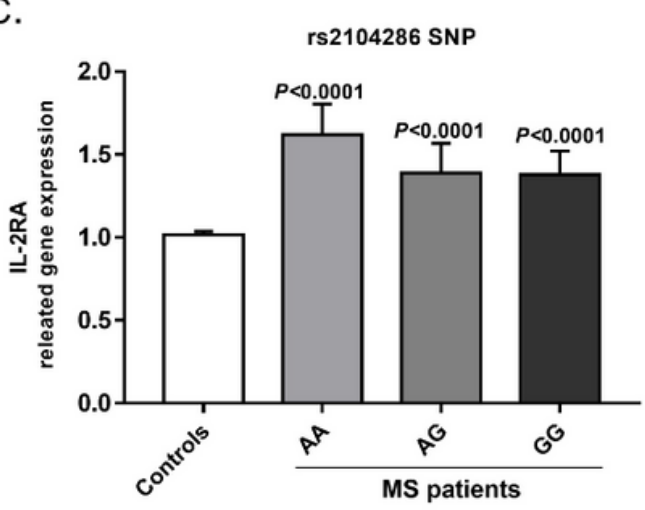

Figure 1

Comparison ILR-2RA gene expression between MS cases and control group 
A.

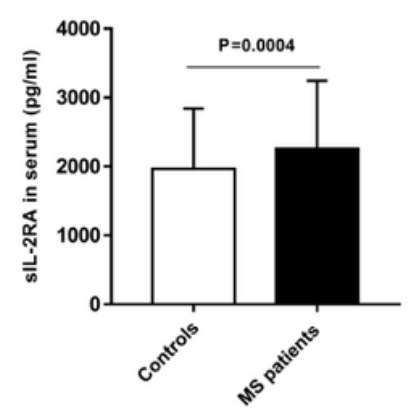

B.

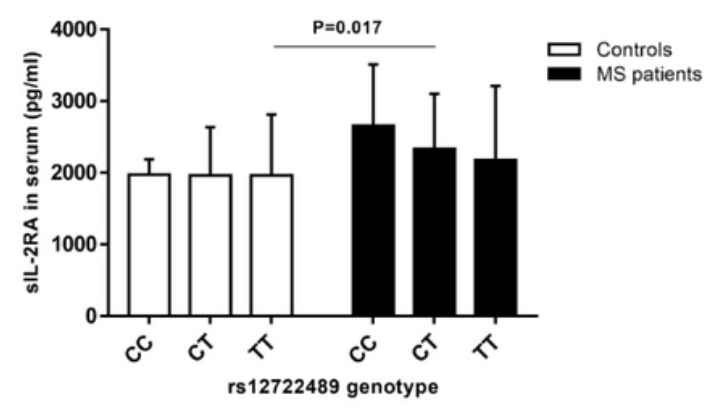

C.

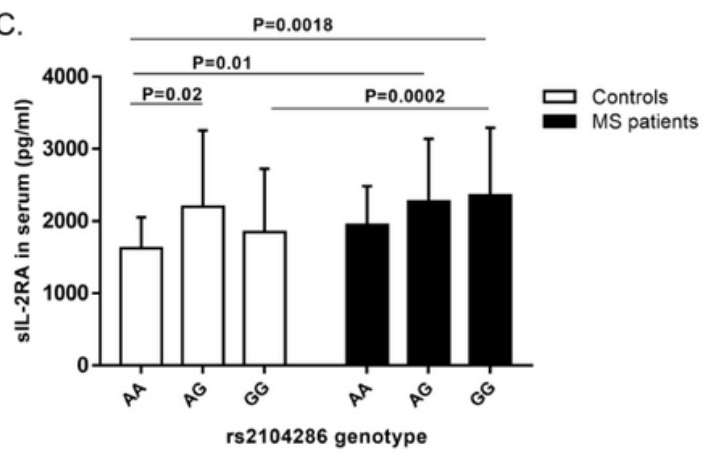

Figure 2

sILR-2RA concentration in the serum of MS patient and healthy subjects 